in vivo $35: 2763-2770(2021)$

doi:10.21873/invivo.12561

\title{
Emotional Problems Prior to Adjuvant Radiation Therapy for Breast Cancer
}

\author{
DIRK RADES ${ }^{1}$, CARLOS A. NARVAEZ ${ }^{1}$, LIESA DZIGGEL ${ }^{1}$, SOEREN TVILSTED ${ }^{2}$, \\ TROELS W. KJAER ${ }^{3}$, STEVEN E. SCHILD ${ }^{4}$ and TOBIAS BARTSCHT ${ }^{5}$ \\ ${ }^{1}$ Department of Radiation Oncology, University of Lübeck, Lübeck, Germany; \\ ${ }^{2}$ Research Projects and Clinical Optimization, Zealand University Hospital, Koege, Denmark; \\ ${ }^{3}$ Neurological Department, Zealand University Hospital, Roskilde, Denmark; \\ ${ }^{4}$ Department of Radiation Oncology, Mayo Clinic, Scottsdale, AZ, U.S.A.; \\ ${ }^{5}$ Department of Hematology and Oncology, University of Lübeck, Lübeck, Germany
}

\begin{abstract}
Background/Aim: Being scheduled for radiotherapy can cause emotional distress. This study aimed to identify risk factors in 338 patients assigned to radiotherapy for breast cancer. Patients and Methods: Nineteen potential risk factors including the COVID-19 pandemic were investigated for associations with the six emotional problems included in the National Comprehensive Cancer Network Distress Thermometer. Results: Worry and fears were significantly associated with age $\leq 60$ years; sadness with age and Karnofsky performance score (KPS) $<90$; depression with KPS and Charlson Comorbidity Index $\geq 3$; loss of interest with KPS. Trends were found for associations between sadness and additional breast cancer/DCIS, Charlson Index and chemotherapy; between depression and additional breast cancer/DCIS, treatment volume and nodal stage N1-3; between nervousness and additional breast cancer/DCIS, mastectomy and triplenegativity; between loss of interest and Charlson Index, family history of breast cancer/DCIS, invasive cancer, chemotherapy, and treatment volume. The COVID-19 pandemic did not increase emotional problems. Conclusion: Several risk factors for emotional problems were identified. Patients with such factors should receive psychological support well before radiotherapy.
\end{abstract}

This article is freely accessible online.

Correspondence to: Prof. Dirk Rades, MD, Department of Radiation Oncology, University of Lübeck, Lübeck, Ratzeburger Allee 160, 23562 Lübeck, Germany. Tel: +49 45150045401, Fax: +49 45150045404, e-mail: dirk.rades@uksh.de

Key Words: Emotional problems, breast cancer, radiation therapy, risk factors, COVID-19 pandemic.
Breast cancer is the most common site of new cancers in women in many countries including Denmark and Germany (1-5). Most patients with primary breast cancer receive adjuvant radiotherapy as an integral part of breast-conserving treatment. Radiotherapy may also be indicated after mastectomy if specific risk factors exist (6). The vast majority of patients assigned to adjuvant irradiation receive external beam radiotherapy (EBRT) of the whole breast with or without a boost to the tumor bed (6). Depending on the nodal stage, the treatment volume of radiotherapy may include the locoregional lymph nodes in addition to the breast.

Being scheduled for radiotherapy and the radiation treatment itself can be associated with emotional distress. Breast cancer patients were reported to have higher prevalence and distress levels than patients with several other malignancies $(7,8)$. According to previous studies, $30-50 \%$ of women with breast cancer report moderate to severe distress and decline in quality of life during their course of radiation treatment (2). Moreover, higher levels of distress prior to surgery or chemotherapy for breast cancer were associated with increased adverse events during and following treatment (2). For patients receiving radiotherapy, it is important to avoid interruption of the treatment due to adverse events in order to achieve optimal results. Very few studies investigated emotional distress before a course of radiotherapy. Browall et al. found that better emotional functioning predicted better quality of live in patients with breast cancer receiving adjuvant radiotherapy (9). Mose et al. reported that $48 \%$ of patients with early-stage breast cancer were afraid of adjuvant radiotherapy after breast-conserving surgery (BCS) (10). In their study, younger age was the only significant predictor of pre-radiotherapy distress. Sohl et al. identified several predictors of pre-radiotherapy emotional distress including cancer stage, upfront mastectomy, response expectations and pessimism (2). Knowledge of such risk factors is important, since patients with these factors may 
experience impairment in quality of life and more pronounced radiation-related adverse events and require early psychological intervention $(2,9)$. Therefore, the present study investigated many patient-, tumor-, and treatment-related factors and a potential impact of the COVID-19 pandemic for associations with emotional problems prior to a course of adjuvant radiotherapy for primary breast cancer.

\section{Patients and Methods}

Data of 338 female patients with breast cancer scheduled for adjuvant treatment with external-beam radiotherapy (EBRT) were evaluated for emotional problems prior to the radiation course. This retrospective study was approved by the responsible Ethics Committee (University of Lübeck, reference 21-178). Patients included in this study had completed a questionnaire regarding distress (National Comprehensive Cancer Network Distress Thermometer) at the time of the pre-radiotherapy consent discussion with the patients (11). Of these patients who were previously evaluated for sleep disorders (12), 163 presented before and 175 patients during the COVID-19 pandemic.

Prior to radiotherapy, all patients had received surgery for their breast tumor, either as BCS $(n=302)$ or mastectomy $(n=36)$. Patients treated with BCS were scheduled for whole-breast irradiation $(15 \times 2.667$ Gy over 3 weeks or $28 \times 1.8$ Gy over 6.5 weeks $)$ plus/minus a boost to the tumor bed $(5 \times 2.0$ Gy over 1 week) (2). Patients scheduled for radiotherapy of the chest wall after mastectomy had specific risk factors and were assigned to EBRT with $28 \times 1.8$ Gy over 6.5 weeks. Sixty-seven patients with involvement for more than three axillary lymph nodes or one to three lymph nodes plus risk factors were scheduled for EBRT of the whole breast plus locoregional lymph node regions (2).

The National Comprehensive Cancer Network Distress Thermometer (11) addresses six emotional problems, namely worry, fears, sadness, depression, nervousness and loss of interest in usual activities. Based on the patients' statements in the distress thermometer evaluation (patient reported outcomes), each problem was categorized as absent (no) or present (yes).

For each of the six emotional problems, 19 potential risk factors were investigated. These factors were relation to the COVID-19 pandemic (before $v s$. during), age ( $\leq 60 v s . \geq 61$ years, median age $=60.5$ years); Karnofsky performance score (KPS 90-100 vs. <90); Charlson Comorbidity Index ( 2 vs. $\geq 3$, median index=2); previous or concurrent breast cancer or ductal carcinoma in situ (DCIS) (no $v s$. yes); previous or concurrent malignancy including breast cancer/DCIS (no vs. yes); family history of malignancy including breast cancer/DCIS (no vs. yes); family history of breast cancer/DCIS (no vs. yes); previous radiotherapy (no vs. yes); primary tumor type (DCIS only vs. invasive breast cancer with/without DCIS); type of surgery for breast tumor (BCS vs. mastectomy); preceding chemotherapy (no vs. yes), start of hormonal therapy (tamoxifen or aromatase inhibitors) before radiotherapy (no vs. yes); and volume of radiation treatment (without $v s$. with lymph nodes). In patients with invasive breast cancer, primary tumor $(\mathrm{T})$ stage (1-2 vs. 3-4), nodal $(\mathrm{N})$ stage $(0-$ $1_{\text {mi }}$ vs. 1-3), distant metastasis (no vs. yes), histologic grading $(\mathrm{G})$ (1-2 vs. 3) and triple (estrogen receptor, progesterone receptor and status of the human epidermal growth factor receptor 2/neu) negativity (no vs. yes) were additionally evaluated.
The statistical analyses regarding associations between emotional problems and potential risk factors were performed with the chisquare test. In the case that the number of patients in a subgroup was fewer than 5, the Fisher's exact test was used. For each of the six emotional problems, separate analyses including the 19 potential risk factors were performed. When applying Bonferroni adjustment for 19 tests, $p$-Values of $<0.0026$ were considered to be significant representing an alpha level of $<0.05 . p$-Values of $<0.05$ were considered as indicating a trend.

\section{Results}

The COVID-19 pandemic had no significant impact on the prevalence of emotional problems (Tables I, II, III, IV, V and VI). A total of 127 patients $(38 \%)$ complained about worry prior to their radiotherapy course. Worry was significantly associated with age $\leq 60$ years $(p<0.0001)$ (Table I). Fears were stated by 154 patients $(46 \%)$ and were significantly associated with age $\leq 60$ years $(p<0.0001)$, too (Table II).

Sadness was reported by 97 patients (29\%). This emotional problem was significantly associated with age $\leq 60$ years $(p=0.0012)$ and a KPS $<90(p=0.0022)$ (Table III). Borderline significance was found for history of previous or concurrent breast cancer/DCIS $(p=0.0056)$, and trends were observed for a Charlson Comorbidity Index $\geq 3 \quad(p=0.038)$ and preceding chemotherapy $(p=0.028)$.

Forty-one patients (12\%) reported depression, which was significantly associated with a KPS $<90(p=0.0017)$ and a Charlson Comorbidity Index $\geq 3$ ( $p=0.0013)$ (Table IV). Trends for associations with depression were found for previous or concurrent breast cancer/DCIS $(p=0.023)$, volume of radiation treatment including locoregional lymph nodes $(p=0.015)$ and nodal stage N1-3 $(p=0.033)$.

Nervousness was stated by 98 patients (29\%). No investigated factor was significantly associated with nervousness, but trends for such an association were found for previous or concurrent breast cancer/DCIS $(p=0.022)$, upfront mastectomy $(p=0.035)$ and triple-negativity $(p=0.042)$ (Table V).

Loss of interest in usual activities was reported by 50 patients $(15 \%)$ and showed a significant association with a KPS $<90(p<0.0001)$ (Table VI). Trends were observed for a Charlson Comorbidity Index $\geq 3(p=0.011)$, family history of breast cancer/DCIS $(p=0.029)$, invasive breast cancer $(p=0.041)$, preceding chemotherapy $(p=0.022)$ and volume of radiation treatment including locoregional lymph nodes $(p=0.021)$.

\section{Discussion}

Most patients with primary non-metastatic breast cancer receive breast-conserving surgery followed by radiotherapy (6). Moreover, selected patients with risk factors for developing a local or locoregional recurrence require 
Table I. Associations of the investigated characteristics and worry (338 patients).

\begin{tabular}{|c|c|c|c|c|}
\hline \multirow[b]{2}{*}{ Characteristic } & & \multicolumn{2}{|c|}{ Worry, n (\%) } & \multirow[b]{2}{*}{$p$-Value } \\
\hline & & No & Yes & \\
\hline \multirow[t]{2}{*}{ COVID-19 pandemic } & Before & $102(63)$ & $61(37)$ & 0.96 \\
\hline & During & $109(62)$ & $66(38)$ & \\
\hline \multirow[t]{2}{*}{ Age } & $\leq 60$ Years & $85(50)$ & $84(50)$ & $<0.0001$ \\
\hline & $\geq 61$ Years & $126(75)$ & $43(25)$ & \\
\hline \multirow{2}{*}{$\begin{array}{l}\text { Karnofsky performance } \\
\text { score }\end{array}$} & $90-100$ & $172(63)$ & $103(37)$ & 0.92 \\
\hline & $<90$ & $39(62)$ & $24(38)$ & \\
\hline \multirow{2}{*}{$\begin{array}{l}\text { Charlson comorbidity } \\
\text { index }\end{array}$} & 2 & $137(63)$ & $80(37)$ & 0.72 \\
\hline & $\geq 3$ & $74(61)$ & 47 (39) & \\
\hline \multirow{2}{*}{$\begin{array}{l}\text { Additional breast } \\
\text { cancer/DCIS }\end{array}$} & No & $194(62)$ & $117(38)$ & 0.95 \\
\hline & Yes & $17(63)$ & $10(37)$ & \\
\hline \multirow{2}{*}{$\begin{array}{l}\text { History of additional } \\
\text { tumor }\end{array}$} & No & $187(62)$ & $115(38)$ & 0.58 \\
\hline & Yes & $24(67)$ & $12(33)$ & \\
\hline \multirow{2}{*}{$\begin{array}{l}\text { Family history } \\
\text { of malignancy }\end{array}$} & No & $75(68)$ & $36(32)$ & 0.17 \\
\hline & Yes & $136(60)$ & $91(40)$ & \\
\hline \multirow{2}{*}{$\begin{array}{l}\text { Family history of } \\
\text { breast cancer/DCIS }\end{array}$} & No & $134(64)$ & $76(36)$ & 0.50 \\
\hline & Yes & $77(60)$ & $51(40)$ & \\
\hline \multirow[t]{2}{*}{ Previous radiotherapy } & No & $192(62)$ & $120(38)$ & 0.24 \\
\hline & Yes & $19(73)$ & $7(27)$ & \\
\hline \multirow[t]{2}{*}{ Tumor type } & DCIS & $23(70)$ & $10(30)$ & 0.36 \\
\hline & $\begin{array}{l}\text { Invasive } \\
\text { cancer }\end{array}$ & $188(62)$ & $117(38)$ & \\
\hline \multirow[t]{2}{*}{ Type of surgery } & BCS & $189(63)$ & $113(37)$ & 0.86 \\
\hline & Mastectomy & $22(61)$ & $14(39)$ & \\
\hline \multirow[t]{2}{*}{ Chemotherapy pre-RT } & No & $138(65)$ & $74(35)$ & 0.19 \\
\hline & Yes & $73(58)$ & $53(42)$ & \\
\hline \multirow{2}{*}{$\begin{array}{l}\text { Hormonal therapy } \\
\text { pre-RT }\end{array}$} & No & $165(63)$ & $95(37)$ & 0.47 \\
\hline & Yes & $46(59)$ & $32(41)$ & \\
\hline \multirow{2}{*}{$\begin{array}{l}\text { Treatment volume } \\
\text { of RT }\end{array}$} & Without LN & $176(65)$ & $95(35)$ & 0.055 \\
\hline & With LN & $35(52)$ & $32(48)$ & \\
\hline \multirow[t]{2}{*}{ Primary tumor stage* } & $\mathrm{T} 1-2$ & $173(63)$ & $103(37)$ & 0.25 \\
\hline & T3-4 & $15(52)$ & $14(48)$ & \\
\hline \multirow[t]{2}{*}{ Nodal stage* } & $\mathrm{N} 0-1_{\mathrm{mi}}$ & $141(64)$ & $80(36)$ & 0.21 \\
\hline & $\mathrm{N} 1-3$ & $47(56)$ & $37(44)$ & \\
\hline \multirow[t]{2}{*}{ Distant metastasis* } & No & $187(62)$ & $115(38)$ & 0.56 \\
\hline & Yes & $1(33)$ & $2(67)$ & \\
\hline \multirow[t]{2}{*}{ Histologic grading* } & G1-2 & $135(63)$ & $78(37)$ & 0.34 \\
\hline & G3 & $53(58)$ & $39(42)$ & \\
\hline \multirow[t]{2}{*}{ Triple negative tumor* } & No & $177(63)$ & $103(37)$ & 0.058 \\
\hline & Yes & $11(44)$ & $14(56)$ & \\
\hline
\end{tabular}

BCS: Breast-conserving surgery; DCIS: ductal carcinoma in situ; LN: lymph nodes; RT: radiotherapy. *Invasive cancer only $(\mathrm{n}=305)$. When applying Bonferroni correction, $p$-values $<0.0026$ are significant and shown in bold.

radiation treatment after skin-sparing or radical mastectomy (6). In addition to diagnosis of breast cancer, just the being scheduled for post-operative irradiation can lead to significant emotional distress. In the study of Mose et al. that included 111 women irradiated with 56 Gy following BCS, $53 \%$ of the patients experienced distress due to the diagnosis of breast cancer and $48 \%$ of the patients felt distressed because of the imminent radiation treatment (10). Moreover,
Table II. Associations of the investigated characteristics and fears (338 patients).

\begin{tabular}{|c|c|c|c|c|}
\hline \multirow[b]{2}{*}{ Characteristic } & & \multicolumn{2}{|c|}{ Fear, n (\%) } & \multirow[b]{2}{*}{$p$-Value } \\
\hline & & No & Yes & \\
\hline \multirow[t]{2}{*}{ COVID-19 pandemic } & Before & $87(53)$ & $76(47)$ & 0.71 \\
\hline & During & $97(55)$ & $78(45)$ & \\
\hline \multirow[t]{2}{*}{ Age } & $\leq 60$ Years & $74(44)$ & $95(56)$ & $<0.0001$ \\
\hline & $\geq 61$ Years & $110(65)$ & $59(35)$ & \\
\hline \multirow{2}{*}{$\begin{array}{l}\text { Karnofsky performance } \\
\text { score score }\end{array}$} & $90-100$ & $152(55)$ & $123(45)$ & 0.52 \\
\hline & $<90$ & $32(51)$ & $31(49)$ & \\
\hline \multirow{2}{*}{$\begin{array}{l}\text { Charlson comorbidity } \\
\text { index }\end{array}$} & 2 & $124(57)$ & $93(43)$ & 0.18 \\
\hline & $\geq 3$ & $60(50)$ & $61(50)$ & \\
\hline \multirow{2}{*}{$\begin{array}{l}\text { Additional breast } \\
\text { cancer/DCIS }\end{array}$} & No & $172(55)$ & $139(45)$ & 0.28 \\
\hline & Yes & $12(44)$ & $15(56)$ & \\
\hline \multirow{2}{*}{$\begin{array}{l}\text { History of additional } \\
\text { tumor }\end{array}$} & No & $163(54)$ & $139(46)$ & 0.62 \\
\hline & Yes & $21(58)$ & $15(42)$ & \\
\hline \multirow{2}{*}{$\begin{array}{l}\text { Family history } \\
\text { of malignancy }\end{array}$} & No & $65(59)$ & $46(41)$ & 0.29 \\
\hline & Yes & $119(52)$ & $108(48)$ & \\
\hline \multirow{2}{*}{$\begin{array}{l}\text { Family history of } \\
\text { breast cancer/DCIS }\end{array}$} & No & $120(57)$ & $90(43)$ & 0.20 \\
\hline & Yes & $64(50)$ & $64(50)$ & \\
\hline \multirow[t]{2}{*}{ Previous radiotherapy } & No & $168(54)$ & $144(46)$ & 0.45 \\
\hline & Yes & $16(62)$ & $10(38)$ & \\
\hline \multirow[t]{2}{*}{ Tumor type } & DCIS & $18(55)$ & $15(45)$ & 0.99 \\
\hline & $\begin{array}{l}\text { Invasive } \\
\text { cancer }\end{array}$ & $166(54)$ & $139(46)$ & \\
\hline \multirow[t]{2}{*}{ Type of surgery } & $\mathrm{BCS}$ & $166(55)$ & $136(45)$ & 0.57 \\
\hline & Mastectomy & $18(50)$ & $18(50)$ & \\
\hline \multirow[t]{2}{*}{ Chemotherapy pre-RT } & No & $117(55)$ & $95(45)$ & 0.72 \\
\hline & Yes & $67(53)$ & $59(47)$ & \\
\hline \multirow{2}{*}{$\begin{array}{l}\text { Hormonal therapy } \\
\text { pre-RT }\end{array}$} & No & $143(55)$ & $117(45)$ & 0.70 \\
\hline & Yes & $41(53)$ & $37(47)$ & \\
\hline \multirow{2}{*}{$\begin{array}{l}\text { Treatment volume } \\
\text { of RT }\end{array}$} & Without LN & $154(57)$ & $117(43)$ & 0.076 \\
\hline & With LN & $30(45)$ & $37(55)$ & \\
\hline \multirow[t]{2}{*}{ Primary tumor stage* } & $\mathrm{T} 1-2$ & $151(55)$ & $125(45)$ & 0.76 \\
\hline & T3-4 & $15(52)$ & $14(48)$ & \\
\hline \multirow[t]{2}{*}{ Nodal stage* } & N0-1 ${ }_{\mathrm{mi}}$ & $126(57)$ & $95(43)$ & 0.14 \\
\hline & $\mathrm{N} 1-3$ & $40(48)$ & $44(52)$ & \\
\hline \multirow[t]{2}{*}{ Distant metastasis* } & No & $165(55)$ & $137(45)$ & 0.59 \\
\hline & Yes & $1(33)$ & $2(67)$ & \\
\hline \multirow[t]{2}{*}{ Histologic grading* } & G1-2 & $118(55)$ & $95(45)$ & 0.60 \\
\hline & G3 & $48(52)$ & $44(48)$ & \\
\hline \multirow[t]{2}{*}{ Triple negative tumor* } & No & $153(55)$ & $127(45)$ & 0.80 \\
\hline & Yes & $13(52)$ & $12(48)$ & \\
\hline
\end{tabular}

BCS: Breast-conserving surgery; DCIS: ductal carcinoma in situ; LN: lymph nodes; RT: radiotherapy. *Invasive cancer only $(\mathrm{n}=305)$. When applying Bonferroni correction, $p$-values $<0.0026$ are significant and shown in bold.

better pre-treatment emotional functioning was reported to be associated with improved overall quality of life at the end of treatment in a series of 75 postmenopausal women receiving adjuvant radiotherapy for breast cancer (9). Therefore, it is important to identify patients who will experience emotional distress prior to the radiotherapy course to provide psychological support timely before starting treatment. Compared to the previous study of Sohl 
Table III. Associations of the investigated characteristics and sadness (338 patients).

\begin{tabular}{|c|c|c|c|c|}
\hline \multirow[b]{2}{*}{ Characteristic } & & \multicolumn{2}{|c|}{ Sadness, n (\%) } & \multirow[b]{2}{*}{$p$-Value } \\
\hline & & No & Yes & \\
\hline \multirow[t]{2}{*}{ COVID-19 pandemic } & Before & $113(69)$ & $50(31)$ & 0.44 \\
\hline & During & $128(73)$ & $47(27)$ & \\
\hline \multirow[t]{2}{*}{ Age } & $\leq 60$ Years & $107(63)$ & $62(37)$ & 0.0012 \\
\hline & $\geq 61$ Years & $134(79)$ & $35(21)$ & \\
\hline \multirow{2}{*}{$\begin{array}{l}\text { Karnofsky performance } \\
\text { score }\end{array}$} & $90-100$ & $206(75)$ & $69(25)$ & 0.0022 \\
\hline & $<90$ & $35(56)$ & $28(44)$ & \\
\hline \multirow{2}{*}{$\begin{array}{l}\text { Charlson comorbidity } \\
\text { index }\end{array}$} & 2 & $163(75)$ & $54(25)$ & 0.038 \\
\hline & $\geq 3$ & $78(64)$ & $43(36)$ & \\
\hline \multirow{2}{*}{$\begin{array}{l}\text { Additional breast } \\
\text { cancer/DCIS }\end{array}$} & No & $228(73)$ & $83(27)$ & 0.0056 \\
\hline & Yes & $13(48)$ & $14(52)$ & \\
\hline \multirow{2}{*}{$\begin{array}{l}\text { History of additional } \\
\text { tumor }\end{array}$} & No & $216(72)$ & $86(28)$ & 0.79 \\
\hline & Yes & $25(69)$ & $11(31)$ & \\
\hline \multirow{2}{*}{$\begin{array}{l}\text { Family history } \\
\text { of malignancy }\end{array}$} & No & $84(76)$ & $27(24)$ & 0.21 \\
\hline & Yes & $157(69)$ & $70(31)$ & \\
\hline \multirow{2}{*}{$\begin{array}{l}\text { Family history of } \\
\text { breast cancer/DCIS }\end{array}$} & No & $150(71)$ & $60(29)$ & 0.95 \\
\hline & Yes & $91(71)$ & 37 (29) & \\
\hline \multirow[t]{2}{*}{ Previous radiotherapy } & No & $224(72)$ & $88(28)$ & 0.49 \\
\hline & Yes & $17(65)$ & $9(35)$ & \\
\hline \multirow[t]{2}{*}{ Tumor type } & DCIS & $26(79)$ & $7(21)$ & 0.32 \\
\hline & $\begin{array}{l}\text { Invasive } \\
\text { cancer }\end{array}$ & $215(70)$ & $90(30)$ & \\
\hline \multirow[t]{2}{*}{ Type of surgery } & BCS & $218(72)$ & $84(28)$ & 0.30 \\
\hline & Mastectomy & $23(44)$ & $13(36)$ & \\
\hline \multirow[t]{2}{*}{ Chemotherapy pre-RT } & No & $160(75)$ & $52(25)$ & 0.028 \\
\hline & Yes & $81(64)$ & $45(36)$ & \\
\hline \multirow{2}{*}{$\begin{array}{l}\text { Hormonal therapy } \\
\text { pre-RT }\end{array}$} & No & $188(72)$ & $72(28)$ & 0.46 \\
\hline & Yes & $53(68)$ & $25(32)$ & \\
\hline \multirow{2}{*}{$\begin{array}{l}\text { Treatment volume } \\
\text { of RT }\end{array}$} & Without LN & $199(73)$ & $72(27)$ & 0.082 \\
\hline & With LN & $42(63)$ & $25(37)$ & \\
\hline \multirow[t]{2}{*}{ Primary tumor stage* } & $\mathrm{T} 1-2$ & $195(71)$ & $81(29)$ & 0.85 \\
\hline & T3-4 & $20(69)$ & $9(31)$ & \\
\hline \multirow[t]{2}{*}{ Nodal stage* } & $\mathrm{N} 0-1_{\mathrm{mi}}$ & $161(73)$ & $60(27)$ & 0.14 \\
\hline & $\mathrm{N} 1-3$ & $54(64)$ & $30(36)$ & \\
\hline \multirow[t]{2}{*}{ Distant metastasis* } & No & $213(71)$ & $89(29)$ & $>0.99$ \\
\hline & Yes & $2(67)$ & $1(33)$ & \\
\hline \multirow[t]{2}{*}{ Histologic grading* } & G1-2 & $156(73)$ & $57(27)$ & 0.11 \\
\hline & G3 & $59(64)$ & $33(36)$ & \\
\hline \multirow{2}{*}{ Triple negative tumor* } & No & $196(70)$ & $84(30)$ & 0.53 \\
\hline & Yes & $19(76)$ & $6(24)$ & \\
\hline
\end{tabular}

BCS: Breast-conserving surgery; DCIS: ductal carcinoma in situ; LN: lymph nodes; RT: radiotherapy. *Invasive cancer only $(\mathrm{n}=305)$. When applying Bonferroni correction, $p$-values $<0.0026$ are significant and shown in bold.

et al., we investigated additional potential risk factors for emotional distress prior to radiotherapy for primary breast cancer. Our study focused on the six emotional problems considered in the National Comprehensive Cancer Network Distress Thermometer, i.e. worry, fears, sadness, depression, nervousness, and loss of interest in usual activities (11).

According to the results of this study, younger age was significantly associated with worry, fears and sadness. Lower
Table IV. Associations of the investigated characteristics and depression (337 patients).

\begin{tabular}{|c|c|c|c|c|}
\hline \multirow[b]{2}{*}{ Characteristic } & & \multicolumn{2}{|c|}{ Depression, n (\%) } & \multirow[b]{2}{*}{$p$-Value } \\
\hline & & No & Yes & \\
\hline \multirow[t]{2}{*}{ COVID-19 pandemic } & Before & $142(87)$ & $21(13)$ & 0.70 \\
\hline & During & $154(89)$ & $20(11)$ & \\
\hline \multirow[t]{2}{*}{ Age } & $\leq 60$ Years & $143(85)$ & $25(15)$ & 0.13 \\
\hline & $\geq 61$ Years & $153(91)$ & $16(9)$ & \\
\hline \multirow{2}{*}{$\begin{array}{l}\text { Karnofsky performance } \\
\text { score }\end{array}$} & $90-100$ & $248(91)$ & $26(9)$ & 0.0017 \\
\hline & $<90$ & $48(76)$ & $15(24)$ & \\
\hline \multirow{2}{*}{$\begin{array}{l}\text { Charlson comorbidity } \\
\text { index }\end{array}$} & 2 & $199(92)$ & $17(8)$ & 0.0013 \\
\hline & $\geq 3$ & $97(80)$ & $24(20)$ & \\
\hline \multirow{2}{*}{$\begin{array}{l}\text { Additional breast } \\
\text { cancer/DCIS }\end{array}$} & No & $276(89)$ & $34(11)$ & 0.023 \\
\hline & Yes & $20(74)$ & $7(26)$ & \\
\hline \multirow{2}{*}{$\begin{array}{l}\text { History of additional } \\
\text { tumor }\end{array}$} & No & $264(88)$ & $37(12)$ & $>0.99$ \\
\hline & Yes & $32(89)$ & $4(11)$ & \\
\hline \multirow{2}{*}{$\begin{array}{l}\text { Family history } \\
\text { of malignancy }\end{array}$} & No & $99(90)$ & $11(10)$ & 0.40 \\
\hline & Yes & $197(87)$ & $30(13)$ & \\
\hline \multirow{2}{*}{$\begin{array}{l}\text { Family history of } \\
\text { breast cancer/DCIS }\end{array}$} & No & $178(85)$ & $31(15)$ & 0.056 \\
\hline & Yes & $118(92)$ & $10(8)$ & \\
\hline \multirow[t]{2}{*}{ Previous radiotherapy } & No & $274(88)$ & $37(12)$ & 0.54 \\
\hline & Yes & $22(85)$ & $4(15)$ & \\
\hline \multirow[t]{2}{*}{ Tumor type } & DCIS & $30(91)$ & $3(9)$ & 0.78 \\
\hline & $\begin{array}{l}\text { Invasive } \\
\text { cancer }\end{array}$ & $266(88)$ & $38(13)$ & \\
\hline \multirow[t]{2}{*}{ Type of surgery } & BCS & $264(88)$ & $37(12)$ & $>0.99$ \\
\hline & Mastectomy & $32(89)$ & $4(11)$ & \\
\hline \multirow[t]{2}{*}{ Chemotherapy pre-RT } & No & $190(90)$ & $21(10)$ & 0.11 \\
\hline & Yes & $106(84)$ & $20(16)$ & \\
\hline \multirow{2}{*}{$\begin{array}{l}\text { Hormonal therapy } \\
\text { pre-RT }\end{array}$} & No & $231(89)$ & $28(11)$ & 0.17 \\
\hline & Yes & $65(83)$ & $13(17)$ & \\
\hline \multirow{2}{*}{$\begin{array}{l}\text { Treatment volume of RT } \\
\text { of RT }\end{array}$} & Without LN & $243(90)$ & $27(10)$ & 0.015 \\
\hline & With LN & $53(79)$ & $14(21)$ & \\
\hline \multirow[t]{2}{*}{ Primary tumor stage* } & $\mathrm{T} 1-2$ & $239(87)$ & $36(13)$ & 0.55 \\
\hline & T3-4 & $27(93)$ & $2(7)$ & \\
\hline \multirow[t]{2}{*}{ Nodal stage* } & $\mathrm{N} 0-1_{\mathrm{mi}}$ & $198(90)$ & $22(10)$ & 0.033 \\
\hline & $\mathrm{N} 1-3$ & $68(81)$ & $16(19)$ & \\
\hline \multirow[t]{2}{*}{ Distant metastasis* } & No & $264(88)$ & $37(12)$ & 0.33 \\
\hline & Yes & $2(67)$ & $1(33)$ & \\
\hline \multirow[t]{2}{*}{ Histologic grading* } & G1-2 & $189(89)$ & $23(11)$ & 0.19 \\
\hline & G3 & $77(84)$ & $15(16)$ & \\
\hline \multirow[t]{2}{*}{ Triple negative tumor* } & No & $243(87)$ & $36(13)$ & 0.75 \\
\hline & Yes & $23(92)$ & $2(8)$ & \\
\hline
\end{tabular}

BCS: Breast-conserving surgery; DCIS: ductal carcinoma in situ; LN: lymph nodes; RT: radiotherapy. *Invasive cancer only $(\mathrm{n}=304)$. When applying Bonferroni correction, $p$-values $<0.0026$ are significant and shown in bold.

(worse) performance score showed significant associations with sadness, depression, and loss of interest in usual activities. Higher comorbidity index was significantly associated with depression and showed trends for associations with sadness and loss of interest in usual activities. Previous or concurrent breast cancer/DCIS was almost significantly associated with sadness, and trends were found for associations with depression and nervousness. 
Table V. Associations of the investigated characteristics and nervousness (338 patients).

\begin{tabular}{|c|c|c|c|c|}
\hline \multirow[b]{2}{*}{ Characteristic } & & \multicolumn{2}{|c|}{ Nervousness, n (\%) } & \multirow[b]{2}{*}{$p$-Value } \\
\hline & & No & Yes & \\
\hline \multirow[t]{2}{*}{ COVID-19 pandemic } & Before & $115(71)$ & 48 (29) & 0.86 \\
\hline & During & $125(71)$ & $50(29)$ & \\
\hline \multirow[t]{2}{*}{ Age } & $\leq 60$ Years & $121(72)$ & $48(28)$ & 0.81 \\
\hline & $\geq 61$ Years & $119(70)$ & $50(30)$ & \\
\hline \multirow{2}{*}{$\begin{array}{l}\text { Karnofsky performance } \\
\text { score }\end{array}$} & $90-100$ & $200(73)$ & $75(27)$ & 0.15 \\
\hline & $<90$ & $40(63)$ & $23(37)$ & \\
\hline \multirow{2}{*}{$\begin{array}{l}\text { Charlson comorbidity } \\
\text { index }\end{array}$} & 2 & $154(71)$ & $63(29)$ & 0.98 \\
\hline & $\geq 3$ & $86(71)$ & $35(29)$ & \\
\hline \multirow{2}{*}{$\begin{array}{l}\text { Additional breast } \\
\text { cancer/DCIS }\end{array}$} & No & $226(73)$ & $85(27)$ & 0.022 \\
\hline & Yes & $14(52)$ & $13(48)$ & \\
\hline \multirow{2}{*}{$\begin{array}{l}\text { History of additional } \\
\text { tumor }\end{array}$} & No & $214(71)$ & $88(29)$ & 0.86 \\
\hline & Yes & $26(72)$ & $10(28)$ & \\
\hline \multirow{2}{*}{$\begin{array}{l}\text { Family history } \\
\text { of malignancy }\end{array}$} & No & $79(71)$ & $32(29)$ & 0.96 \\
\hline & Yes & $161(71)$ & $66(29)$ & \\
\hline \multirow{2}{*}{$\begin{array}{l}\text { Family history of breast } \\
\text { cancer/DCIS }\end{array}$} & No & $147(70)$ & $63(30)$ & 0.60 \\
\hline & Yes & $93(73)$ & $35(27)$ & \\
\hline \multirow[t]{2}{*}{ Previous radiotherapy } & No & $225(72)$ & $87(28)$ & 0.12 \\
\hline & Yes & $15(58)$ & $11(42)$ & \\
\hline \multirow[t]{2}{*}{ Tumor type } & DCIS & $26(79)$ & $7(21)$ & 0.30 \\
\hline & $\begin{array}{l}\text { Invasive } \\
\text { cancer }\end{array}$ & $214(70)$ & $91(30)$ & \\
\hline \multirow[t]{2}{*}{ Type of surgery } & $\mathrm{BCS}$ & 209 (69) & $93(31)$ & 0.035 \\
\hline & Mastectomy & $31(86)$ & $5(14)$ & \\
\hline \multirow[t]{2}{*}{ Chemotherapy pre-RT } & No & $148(70)$ & $64(30)$ & 0.53 \\
\hline & Yes & $92(73)$ & $34(27)$ & \\
\hline \multirow{2}{*}{$\begin{array}{l}\text { Hormonal therapy } \\
\text { pre-RT }\end{array}$} & No & $186(72)$ & $74(28)$ & 0.69 \\
\hline & Yes & $54(69)$ & $24(31)$ & \\
\hline \multirow{2}{*}{$\begin{array}{l}\text { Treatment volume } \\
\text { of RT }\end{array}$} & Without LN & $191(70)$ & $80(30)$ & 0.67 \\
\hline & With LN & $49(73)$ & $18(27)$ & \\
\hline \multirow[t]{2}{*}{ Primary tumor stage* } & $\mathrm{T} 1-2$ & $189(68)$ & $87(32)$ & 0.055 \\
\hline & $\mathrm{T} 3-4$ & $25(86)$ & $4(14)$ & \\
\hline \multirow[t]{2}{*}{ Nodal stage* } & No- $1_{\mathrm{mi}}$ & $154(70)$ & $67(30)$ & 0.77 \\
\hline & $\mathrm{N} 1-3$ & $60(71)$ & $24(29)$ & \\
\hline \multirow[t]{2}{*}{ Distant metastasis* } & No & $212(70)$ & $90(30)$ & $>0.99$ \\
\hline & Yes & $2(67)$ & $1(33)$ & \\
\hline \multirow[t]{2}{*}{ Histologic grading* } & G1-2 & $148(69)$ & $65(31)$ & 0.69 \\
\hline & G3 & $66(72)$ & $26(28)$ & \\
\hline \multirow[t]{2}{*}{ Triple negative tumor* } & No & $192(69)$ & $88(31)$ & 0.042 \\
\hline & Yes & $22(88)$ & $3(12)$ & \\
\hline
\end{tabular}

BCS: Breast-conserving surgery; DCIS: ductal carcinoma in situ; LN: lymph nodes; RT: radiotherapy. *Invasive cancer only $(\mathrm{n}=305)$.

Moreover, trends were observed for associations of preceding chemotherapy with sadness and loss of interest in usual activities, and of treatment volumes of radiotherapy including lymph nodes with depression and loss of interest in usual activities. Additional trends were found for associations between nodal stage 1-3 and depression, between, mastectomy and nervousness, between triplenegativity and nervousness, between family history of breast cancer/DCIS and loss of interest in usual activities, and
Table VI. Associations of the investigated characteristics and loss of interest in usual activities (336 patients).

\begin{tabular}{|c|c|c|c|c|}
\hline \multirow[b]{2}{*}{ Characteristic } & & \multicolumn{2}{|c|}{$\begin{array}{l}\text { Loss of interest in } \\
\text { usual activities, n (\%) }\end{array}$} & \multirow[b]{2}{*}{$p$-Value } \\
\hline & & No & Yes & \\
\hline \multirow[t]{2}{*}{ COVID-19 pandemic } & Before & $137(84)$ & $26(16)$ & 0.59 \\
\hline & During & 149 (86) & $24(14)$ & \\
\hline \multirow[t]{2}{*}{ Age } & $\leq 60$ Years & $140(84)$ & $27(16)$ & 0.51 \\
\hline & $\geq 61$ Years & $146(86)$ & $23(14)$ & \\
\hline \multirow{2}{*}{$\begin{array}{l}\text { Karnofsky performance } \\
\text { score }\end{array}$} & $90-100$ & $243(89)$ & $30(11)$ & $<0.0001$ \\
\hline & $<90$ & $43(68)$ & $20(32)$ & \\
\hline \multirow{2}{*}{$\begin{array}{l}\text { Charlson comorbidity } \\
\text { index }\end{array}$} & 2 & $191(89)$ & $24(11)$ & 0.011 \\
\hline & $\geq 3$ & $95(79)$ & $26(21)$ & \\
\hline \multirow{2}{*}{$\begin{array}{l}\text { Additional breast } \\
\text { cancer/DCIS }\end{array}$} & No & $265(86)$ & $44(14)$ & 0.26 \\
\hline & Yes & $21(78)$ & $6(22)$ & \\
\hline \multirow{2}{*}{$\begin{array}{l}\text { History of additional } \\
\text { tumor }\end{array}$} & No & $257(86)$ & $43(14)$ & 0.42 \\
\hline & Yes & $29(81)$ & $7(19)$ & \\
\hline \multirow{2}{*}{$\begin{array}{l}\text { Family history } \\
\text { of malignancy }\end{array}$} & No & $96(87)$ & $14(13)$ & 0.44 \\
\hline & Yes & $190(84)$ & $36(16)$ & \\
\hline \multirow{2}{*}{$\begin{array}{l}\text { Family history of breast } \\
\text { cancer/DCIS }\end{array}$} & No & $171(82)$ & $38(18)$ & 0.029 \\
\hline & Yes & $115(91)$ & $12(9)$ & \\
\hline \multirow[t]{2}{*}{ Previous radiotherapy } & No & $265(85)$ & $45(15)$ & 0.52 \\
\hline & Yes & $21(81)$ & $5(19)$ & \\
\hline \multirow[t]{2}{*}{ Tumor type } & DCIS & $32(97)$ & $1(3)$ & 0.041 \\
\hline & $\begin{array}{l}\text { Invasive } \\
\text { cancer }\end{array}$ & $254(84)$ & $49(16)$ & \\
\hline \multirow[t]{2}{*}{ Type of surgery } & $\mathrm{BCS}$ & $257(86)$ & $43(14)$ & 0.42 \\
\hline & Mastectomy & y $29(81)$ & $7(19)$ & \\
\hline \multirow[t]{2}{*}{ Chemotherapy pre-RT } & No & $186(89)$ & $24(11)$ & 0.022 \\
\hline & Yes & $100(79)$ & $26(21)$ & \\
\hline \multirow{2}{*}{$\begin{array}{l}\text { Hormonal therapy } \\
\text { pre-RT }\end{array}$} & No & $221(85)$ & $38(15)$ & 0.84 \\
\hline & Yes & $65(84)$ & $12(16)$ & \\
\hline \multirow{2}{*}{$\begin{array}{l}\text { Treatment volume } \\
\text { of RT }\end{array}$} & Without LN & J 235 (87) & $34(13)$ & 0.021 \\
\hline & With LN & $51(76)$ & $16(24)$ & \\
\hline \multirow[t]{2}{*}{ Primary tumor stage* } & $\mathrm{T} 1-2$ & $230(84)$ & $44(16)$ & 0.87 \\
\hline & T3-4 & $24(83)$ & $5(17)$ & \\
\hline \multirow[t]{2}{*}{ Nodal stage* } & $\mathrm{N} 0-1_{\mathrm{mi}}$ & $188(86)$ & $31(14)$ & 0.12 \\
\hline & $\mathrm{N} 1-3$ & $66(79)$ & $18(21)$ & \\
\hline \multirow[t]{2}{*}{ Distant metastasis* } & No & $252(84)$ & $48(16)$ & 0.41 \\
\hline & Yes & $2(67)$ & $1(33)$ & \\
\hline \multirow[t]{2}{*}{ Histologic grading* } & G1-2 & $178(84)$ & $33(16)$ & 0.70 \\
\hline & G3 & $76(83)$ & $16(17)$ & \\
\hline \multirow[t]{2}{*}{ Triple negative tumor* } & No & $233(84)$ & $45(16)$ & $>0.99$ \\
\hline & Yes & $21(84)$ & $4(16)$ & \\
\hline
\end{tabular}

BCS: Breast-conserving surgery; DCIS: ductal carcinoma in situ; LN: lymph nodes; RT: radiotherapy. *Invasive cancer only $(\mathrm{n}=303)$. When applying Bonferroni correction, $p$-values $<0.0026$ are significant and shown in bold.

between invasive cancer and loss of interest in usual activities. During the interpretation of these results, the retrospective study design should be kept in mind. Retrospective studies always bear the risk of hidden selection biases. However, since a major goal of this study is the comparison between patients who presented before and patients who presented during the COVID-19 pandemic, the 
study can currently not be performed as a prospective trial.

In previous studies investigating pre-radiotherapy emotional distress, younger age, cancer stage and mastectomy were identified as risk factors $(2,10)$. Mose et al. observed high distress levels in women being 58 years or younger (10), and Sohl et al. found significant associations between emotional upset and cancer stage or mastectomy (2). Some other predictors of emotional distress identified in our study were previously reported in different settings. In several studies, younger age was identified as predictor of distress. In 2011, Luutonen et al. presented the results of 276 patients who completed a questionnaire regarding distress, depression and unmet needs during a course of adjuvant radiotherapy for breast cancer (13). Age $<53$ years was an independent predictor of distress $(p=0.001)$. In a cohort of 343 women with newly diagnosed breast cancer who completed the distress thermometer younger women $(<50$ years) experienced higher distress levels (14). In 2016, the results of a prospective observational study from The Netherlands were presented (15). This study included patients with breast cancer regardless of the type of treatment and evaluated clinical distress at 6 and 15 months after first diagnosis of cancer. On univariate analysis, younger age predicted enduring clinical distress (odds ratio=0.96). Moreover, in a systematic review of 42 studies, younger age was confirmed as predictor of distress in female breast cancer survivors (16). In a population-based cohort study following 16,495 women with newly diagnosed breast cancer, younger age was associated with distress during both the phase of hospital-based treatment and transitional survivorship (17).

The prognostic role of the performance status was also previously reported. In the prospective study from The Netherlands, lack of muscle strength, which generally means an impairment of the performance status, was a significant (odds ratio=1.82) predictor of enduring clinical distress (15). In the systematic review of Syrowatka et al. about female breast cancer survivors, lower physical activity, lower quality of physical health, and limitations in physical functioning were associated with distress (16). Moreover, in a surveybased study of 125 female patients with any stage of breast cancer recruited within five years of diagnosis, physical symptom burden was associated with depression, anxiety and distress (18). Associations between co-morbidity and distress were also previously described. In the prospective study from the Netherlands, having two or more co-morbidities was a significant (odds ratio=1.51) predictor of enduring clinical distress (15). In the systematic review of 42 studies, co-morbidities were associated with an increased risk of distress (16). So was rheumatologic disease in the population-based cohort study of Syrowatka et al. (17).

Chemotherapy was identified as predictor of distress in several reports including the Dutch prospective study
$(\mathrm{OR}=2.47)$ and the systematic review of 42 studies $(15,16$, 19). More recently, results of a prospective study of 50 women with breast cancer were presented, in whom a significant increase in fatigue $(p<0.001)$ and psychological distress $(p<0.001)$ was observed when comparing post-chemotherapy results to baseline (prior to chemotherapy) (20). Moreover, the fact that mastectomy (compared to BCS) was associated with increased distress was reported in a prospective study from The Netherlands $(\mathrm{OR}=1.72)$ and in the systematic review of Syrowatka et al. $(15,16,19)$. In addition, patients with any type of cancer who received or were scheduled for surgery experienced significantly more emotional distress than other patients in the study of Thomas et al. (21).

Furthermore, negative hormone receptor status, which is similar to triple-negativity in the present study, was a risk factor of distress in the study of Luutonen et al. (13). And more advanced cancer stage at the time of the first diagnosis was identified as predictor of distress in the systematic review of Syrowatka et al. (15). A more advanced nodal stage requires additional radiotherapy of locoregional lymph nodes, which showed a trend for more emotional distress in the present study. Moreover, irradiation of the locoregional lymph nodes is associated with a risk of additional late sequelae including lymphedema and brachial plexopathy, which likely contributes to higher levels of emotional distress. Invasive cancer also showed a trend toward more emotional distress in this study when compared to DCIS alone. This can be explained by the less favorable prognoses of invasive cancers. Moreover, trends were found for experiencing emotional distress in case of a positive own or family history of breast cancer/DCIS. These findings are supported by previous studies showing that the fear of recurrent breast cancer, which likely applies also to fear of second (contralateral) breast cancer, is an important issue and can cause significant distress and have a negative impact on quality of life (22-24). Moreover, being reminded of a relative's breast cancer disease will likely contribute to emotional distress.

In conclusion, the present study contributes to identification of patients scheduled for radiotherapy of primary breast cancer who have a high risk of emotional distress. This applies particularly to younger patients, patients with a poorer performance status, and patients with a higher comorbidity index. In addition, patients who received a mastectomy or preceding chemotherapy, patients with more advanced or aggressive tumors, and patients with own or family history of breast cancer/DCIS have an increased risk of emotional distress. All of these patients should receive psychological support as soon as possible and optimally before the start of their radiotherapy course. In contrast to the identified risk factors, the COVID-19 pandemic was not associated with an increased prevalence of emotional problems. 


\section{Conflicts of Interest}

On behalf of all Authors, the corresponding Author states that there are no conflicts of interest related to this study.

\section{Authors' Contributions}

D.R., S.T., T.W.K., S.E.S. and T.B. participated in the design of the study. D.R., C.A.N. and L.D. provided the data, which were analyzed by D.R. The article drafted by D.R., S.E.S. and T.B. was reviewed and finally approved by all Authors.

\section{Acknowledgements}

The study was funded by the European Regional Development Fund through the Interreg Deutschland-Danmark program as part of the NorDigHealth project.

\section{References}

1 Siegel RL, Miller KD, Fuchs HE and Jemal A: Cancer statistics, 2021. CA Cancer J Clin 71(1): 7-33, 2021. PMID: 33433946. DOI: $10.3322 /$ caac. 21654

2 Sohl SJ, Schnur JB, Sucala M, David D, Winkel G and Montgomery GH: Distress and emotional well-being in breast cancer patients prior to radiotherapy: an expectancy-based model. Psychol Health 27(3): 347-361, 2012. PMID: 21678183. DOI: $10.1080 / 08870446.2011 .569714$

3 Park JH, Chun M, Jung YS and Bae SH: Predictors of psychological distress trajectories in the first year after a breast cancer diagnosis. Asian Nurs Res (Korean Soc Nurs Sci) 11(4): 268-275, 2017. PMID: 29290274. DOI: 10.1016/j.anr.2017.10.003

4 Danckert B, Ferlay J, Engholm G, Hansen HL, Johannesen TB, Khan S, Køtlum JE, Ólafsdóttir E, Schmidt LKH, Virtanen A and Storm HH: NORDCAN: Cancer incidence, mortality, prevalence and survival in the Nordic Countries, version 8.2. (26.03.2019). Association of the Nordic Cancer Registries. Danish Cancer Society, 2019. Available at: https://www.ancr.nu [Last accessed on May 24, 2021]

5 Katalinic A, Pritzkuleit R and Waldmann A: Recent trends in breast cancer incidence and mortality in Germany. Breast Care (Basel) 4(2): 75-80, 2009. PMID: 20847883. DOI: 10.1159/ 000211526

6 Leitlinienprogramm Onkologie (Deutsche Krebsgesellschaft, Deutsche Krebshilfe, AWMF): S3-Leitlinie Früherkennung, Diagnose, Therapie und Nachsorge des Mammakarzinoms, Version 4.3, 2020. Available at: https://www.leitlinienprogrammonkologie.de/leitlinien/mammakarzinom/ [Last accessed on June 17, 2021]

7 Duan Y, Wang L, Sun Q, Liu X, Ding S, Cheng Q, Xie J and Cheng ASK: Prevalence and determinants of psychological distress in adolescent and young adult patients with cancer: a multicenter survey. Asia Pac J Oncol Nurs 8(3): 314-321, 2021. PMID: 33850965. DOI: 10.4103/2347-5625.311005

8 Herschbach P, Keller M, Knight L, Brandl T, Huber B, Henrich $\mathrm{G}$ and Marten-Mittag B: Psychological problems of cancer patients: a cancer distress screening with a cancer-specific questionnaire. Br J Cancer 91(3): 504-511, 2004. PMID: 15238979. DOI: $10.1038 /$ sj.bjc.6601986
9 Browall M, Ahlberg K, Karlsson P, Danielson E, Persson LO and Gaston-Johansson F: Health-related quality of life during adjuvant treatment for breast cancer among postmenopausal women. Eur J Oncol Nurs 12(3): 180-189, 2008. PMID: 18343197. DOI: 10.1016/j.ejon.2008.01.005

10 Mose S, Budischewski KM, Rahn AN, Zander-Heinz AC, Bormeth S and Böttcher HD: Influence of irradiation on therapyassociated psychological distress in breast carcinoma patients. Int J Radiat Oncol Biol Phys 51(5): 1328-1335, 2001. PMID: 11728694. DOI: 10.1016/s0360-3016(01)01711-4

11 Holland JC, Andersen B, Breitbart WS, Buchmann LO, Compas B, Deshields TL, Dudley MM, Fleishman S, Fulcher CD, Greenberg DB, Greiner CB, Handzo GF, Hoofring L, Hoover C, Jacobsen PB, Kvale E, Levy MH, Loscalzo MJ, McAllister-Black R, Mechanic KY, Palesh O, Pazar JP, Riba MB, Roper K, Valentine AD, Wagner LI, Zevon MA, McMillian NR and Freedman-Cass DA: Distress management. J Natl Compr Canc Netw 11(2): 190209, 2013. PMID: 23411386. DOI: 10.6004/jncen.2013.0027

12 Rades D, Narvaez CA, Schild SE, Tvilsted S and Kjaer TW: Sleep disorders before and during the COVID-19 pandemic in patients assigned to adjuvant radiotherapy for breast cancer. In Vivo 35: 2253-2260, 2021. DOI: 10.21873/invivo.12498

13 Luutonen S, Vahlberg T, Eloranta S, Hyväri H and Salminen E: Breast cancer patients receiving postoperative radiotherapy: distress, depressive symptoms and unmet needs of psychosocial support. Radiother Oncol 100(2): 299-303, 2011. PMID: 21316782. DOI: 10.1016/j.radonc.2011.01.014

14 Mertz BG, Bistrup PE, Johansen C, Dalton SO, Deltour I, Kehlet $\mathrm{H}$ and Kroman N: Psychological distress among women with newly diagnosed breast cancer. Eur J Oncol Nurs 16(4): 439443, 2012. PMID: 22036771. DOI: 10.1016/j.ejon.2011.10.001

15 Lo-Fo-Wong DN, de Haes HC, Aaronson NK, van Abbema DL, den Boer MD, van Hezewijk M, Immink M, Kaptein AA, Menke-Pluijmers MB, Reyners AK, Russell NS, Schriek M, Sijtsema S, van Tienhoven G and Sprangers MA: Predictors of enduring clinical distress in women with breast cancer. Breast Cancer Res Treat 158(3): 563-572, 2016. PMID: 27417105. DOI: $10.1007 / \mathrm{s} 10549-016-3896-7$

16 Syrowatka A, Motulsky A, Kurteva S, Hanley JA, Dixon WG, Meguerditchian AN and Tamblyn R: Predictors of distress in female breast cancer survivors: a systematic review. Breast Cancer Res Treat 165(2): 229-245, 2017. PMID: 28553684. DOI: $10.1007 / \mathrm{s} 10549-017-4290-9$

17 Syrowatka A, Hanley JA, Weir DL, Dixon WG, Meguerditchian AN and Tamblyn R: Ability to predict new-onset psychological distress using routinely collected health data: a population-based cohort study of women diagnosed with breast cancer. J Natl Compr Canc Netw 16(9): 1065-1073, 2018. PMID: 30181418. DOI: $10.6004 /$ jnccn.2018.7038

18 McFarland DC, Shaffer KM, Tiersten A and Holland J: Physical symptom burden and its association with distress, anxiety, and depression in breast cancer. Psychosomatics 59(5): 464-471, 2018. PMID: 29525522. DOI: 10.1016/j.psym.2018.01.005

19 Lo-Fo-Wong DNN, de Haes HCJM, Aaronson NK, van Abbema DL, den Boer MD, van Hezewijk M, Immink M, Kaptein AA, Menke-Pluijmers MBE, Reyners AKL, Russell NS, Schriek M, Sijtsema S, van Tienhoven G, Verdam MGE and Sprangers MAG: Risk factors of unmet needs among women with breast cancer in the post-treatment phase. Psychooncology 29(3): 539549, 2020. PMID: 31785043. DOI: 10.1002/pon.5299 
20 Oh PJ and Cho JR: Changes in fatigue, psychological distress, and quality of life after chemotherapy in women with breast cancer: a prospective study. Cancer Nurs 43(1): E54-E60, 2020. PMID: 30601265. DOI: 10.1097/NCC.0000000000000689

21 Thomas BC, NandaMohan V, Nair MK and Pandey M: Gender, age and surgery as a treatment modality leads to higher distress in patients with cancer. Support Care Cancer 19(2): 239-250, 2010. PMID: 20174987. DOI: 10.1007/s00520-009-0810-4

22 Kenne Sarenmalm E, Ohlén J, Jonsson T and Gaston-Johansson F: Coping with recurrent breast cancer: predictors of distressing symptoms and health-related quality of life. J Pain Symptom Manage 34(1): 24-39, 2007. PMID: 17544244. DOI: 10.1016/ j.jpainsymman.2006.10.017

23 Martínez Arroyo O, Andreu Vaíllo Y, Martínez López P and Galdón Garrido MJ: Emotional distress and unmet supportive care needs in survivors of breast cancer beyond the end of primary treatment. Support Care Cancer 27(3): 1049-1057, 2019. PMID: 30094729. DOI: 10.1007/s00520-018-4394-8
24 Johns SA, Stutz PV, Talib TL, Cohee AA, Beck-Coon KA, Brown LF, Wilhelm LR, Monahan PO, LaPradd ML, Champion VL, Miller KD and Giesler RB: Acceptance and commitment therapy for breast cancer survivors with fear of cancer recurrence: A 3-arm pilot randomized controlled trial. Cancer 126(1): 211-218, 2020. PMID: 31539169. DOI: 10.1002/ cncr.32518

Received May 27, 2021

Revised June 11, 2021

Accepted June 17, 2021 